\title{
Seed-Associated Fungal Diversity and the Molecular Identification of Fusarium with Potential Threat to Ginseng (Panax ginseng) in China
}

\author{
Yi Ming Guan, ${ }^{1,2}$ Jin Chao Deng, ${ }^{1}$ Ying Ying Ma, ${ }^{1}$ Yu $\mathrm{Li}^{2, \dagger}$ and Ya Yu Zhang ${ }^{1, \dagger}$ \\ ${ }^{1}$ Institute of Special Wild Economic Animal and Plant Science, Chinese Academy of Agricultural Sciences, Changchun, Jilin, \\ 130112, China \\ ${ }^{2}$ Engineering Research Center of Chinese Ministry of Education for Edible and Medicinal Fungi, Jilin Agricultural University, \\ Changchun, Jilin, 130118, China
}

\begin{abstract}
The utility of traditional methods for detecting seed-borne fungi is limited by the fact some fungi are unculturable or difficult to isolate. The seed-borne pathogens affecting Panax ginseng cultivation have not been fully characterized. Seed-borne fungi can be identified based on the highthroughput sequencing of internal transcribed spacer (ITS) amplicons. A hierarchical clustering tree diagram analysis based on operational taxonomic units revealed a relationship between the seed-borne fungi and the region from which the seeds were collected. This study analyzed the fungal diversity on 30 ginseng seed samples from the main ginsengproducing areas of China. The 50 most abundant genera were identified including those responsible for ginseng diseases, Fusarium, Alternaria,

species, which are the primary causes of root rot, were detected in all seed samples. The results of a phylogenetic analysis indicated that the seedborne fungal species originating from the same region were closely related. Fungi on ginseng seeds from eight different regions were divided into eight clades, suggesting they were correlated with the local storage medium. A total of 518 Fusarium isolates were obtained and 10 species identified, all of which can be detrimental to ginseng production. Pathogenicity tests proved that seed-borne Fusarium species can infect ginseng seedlings and 2-year-old ginseng root, with potentially adverse effects on ginseng yield and quality.
\end{abstract} Nectria, Coniothyrium, Verticillium, Phoma, and Rhizoctonia. Fusarium
Keywords: fungal diversity, Fusarium, Panax ginseng, seed-borne
Panax ginseng C. A. Meyer is an important medicinal plant that is known worldwide and is used in pharmaceutical and health products as well as in cosmetics (Wang 2001). In terms of cultivated areas, China and Korea are the largest global producers of ginseng.

Unhealthy seeds not only affect vitality and germination but also represent a key factor in the treatment of soil-borne diseases because seeds can be an important inoculum source (Ziezold et al. 1998). After processing their harvested ginseng seeds, farmers store them in media, such as sandy soil, and bury them underground at low temperatures, and they become cracked after they ripen physiologically and morphologically. Maturing and harvested ginseng seeds can carry pathogens, such as Fusarium spp., Ilyonectria spp., Alternaria spp., and Rhizoctonia solani (Guan et al. 2010; Howard et al. 1994; Lee et al. 1981; Reeleder and Fisher 1995; Zhang et al. 1989) and may also be infected by pathogens present in the storage medium. These pathogens affect seed germination and may inhibit

${ }^{\dagger}$ Corresponding authors: Y. Li; fungi966@126.com

and Y. Y. Zhang; zyy1966999@ sina.com

Funding: This study was supported by the Key project at central government level: The ability establishment of sustainable use for valuable Chinese medicine resources (2060302), Collaborative Innovation Project of Science and Technology Innovation Project of Chinese Academy of Agricultural Sciences (Agricultural Science and Technology Innovation Program, 2018XTCX01), National Natural Science Foundation of China (31701354), China Agriculture Research System (CARS-21), Central Public-interest Scientific Institution Basal Research Foundation of Chinese Academy of Agricultural Sciences (1610342016010), and Science and Technology Development Project (20140204056YY, 20191001021XH).

*The $\boldsymbol{e}$-Xtra logo stands for "electronic extra" and indicates that one supplementary figure is published online.

The author(s) declare no conflict of interest.

Accepted for publication 9 September 2019

(C) 2020 The American Phytopathological Society seedling growth. Bunkina (1960) isolated Fusarium martii var. mi$n u s$ and $F$. sporotrichioides var. minus from Korean ginseng seeds. Ziezold et al. (1998) reported that $F$. rostrum, $F$. oxysporum, $F$. solani, and $F$. avenaceum were detected in 22.2, 9.0, 9.0, and $0.9 \%$ of American ginseng embryos, respectively, and also described the effects of these fungi on seed activities. In Korea, Japan, and Canada, Pectobacterium carotovorum, Pseudomonas fluorescens, and Fusarium species, which commonly exist in soils or in plant residues as saprophytes, were isolated from decayed seeds (Howard et al. 1994). These organisms can cause multi-infection in tissues infected by primary intruders. F. equiseti isolated in regions where American ginseng is cultivated was confirmed to cause ginseng root-surface discoloration, and the inoculation of straw with $F$. equiseti resulted in seed infections (Punja et al. 2008). Moreover, F. equiseti was isolated from flowers, berries, and harvested seeds (Punja et al. 2008). Earlier investigations revealed that $F$. solani, $F$. oxysporum, and $F$. equiseti can cause seed decay and seedling damping-off (Howard et al. 1994; Punja 1997). Talaromyces flavus isolated from the ginseng seed coat antagonizes the main pathogens of ginseng, including Ilyonectria destructans, F. oxysporum, R. solani, Sclerotinia nivalis, Botrytis cinerea, and Phytophthora capsici, and directly affects seed dehiscence (Kim et al. 2017).

The main genetic loci currently used for the molecular identification of Fusarium species include rDNA internal transcribed spacer (ITS), translation elongation factor 1-alpha (TEF- $1 \alpha), \beta$-tubulin (BT), mitochondrial small subunit $\mathrm{rDNA}$, phosphate permease, and putative reductase (Glass and Donaldson 1995; O'Donnell et al. 2000). Moreover, TEF- $1 \alpha$ is widely recognized as a DNA barcode for identifying Fusarium species. Some Fusarium species can be identified via the phylogenetic analysis of a single gene sequence. However, most Fusarium species require a phylogenetic analysis involving multiple genes. A Fusarium gene database, which contains the sequences of multiple genes, was established in 2004 (Geiser et al. 2004). Leslie and Summerell (2006) discussed about 70 Fusarium species, and many more have been described in recent years.

The purpose of the study is to determine that ginseng seeds carried a variety of fungal communities associated with important ginseng diseases, especially the threat posed by Fusarium to ginseng cultivation. 


\section{Materials and Methods}

Extraction and amplification of DNA from seed-borne fungi. Ginseng seeds were collected from 30 sampling locations in Jilin Province, which is the major ginseng-producing area of China. The samples were divided into eight groups as outlined in Table 1. Group A samples were stored in a pot with no media at $4^{\circ} \mathrm{C}$, and group B to $\mathrm{H}$ samples from the same administrative counties were stored in different types of media, and they are all mature seeds with a crack. Group C contains samples from two nearby counties. For each sample, $20 \mathrm{~g}$ of seeds were rinsed with sterile water and separated into seed coats and seed kernels for adequate fungal exposure along the crack. They were then placed in 50-ml centrifuge tubes containing $20 \mathrm{ml}$ of sterile water and subjected to sonic oscillation for $20 \mathrm{~min}$ to collect seed-borne fungi. After centrifuging at $12,000 \times g$ for 5 min, DNA was extracted from the sediment using the PowerSoil DNA Isolation Kit (MO BIO, Carlsbad, CA). The quality and concentration of the extracted DNA were evaluated using $1 \%$ agarose gel electrophoresis and a NanoDrop ND-1000 UV spectrophotometer (NanoDrop Technologies, Wilmington, DE). Three replicates were done for each sample.

Table 1. Seed samples collected during this study

\begin{tabular}{|c|c|c|c|c|}
\hline Group & Number & Code & Origin & Storage medium $^{\mathrm{a}}$ \\
\hline \multirow[t]{5}{*}{ A } & 1 & CK1 & Tonghua county & - \\
\hline & 2 & CK2 & Fusong county & - \\
\hline & 3 & CK3 & Jian county & - \\
\hline & 4 & CK4 & Dunhua county & - \\
\hline & 5 & CK5 & Yanji city & - \\
\hline \multirow[t]{3}{*}{ B } & 6 & TH1 & Tonghua county & Sand \\
\hline & 7 & $\mathrm{TH} 2$ & Tonghua county & Sand \\
\hline & 8 & TH3 & Tonghua county & Loess \\
\hline \multirow[t]{4}{*}{$\mathrm{C}$} & 9 & DH1 & Dunhua county & Sand \\
\hline & 10 & $\mathrm{DH} 2$ & Dunhua county & Loess \\
\hline & 11 & JH1 & Jiaohe county & Sand \\
\hline & 12 & $\mathrm{JH} 2$ & Jiaohe county & Sand \\
\hline \multirow[t]{3}{*}{$\mathrm{D}$} & 13 & YJ1 & Yanji city & Sand \\
\hline & 14 & YJ2 & Yanji city & Loess \\
\hline & 15 & YJ3 & Yanji city & Sand \\
\hline \multirow[t]{4}{*}{$\mathrm{E}$} & 16 & FS1 & Southern Fusong county & Sand \\
\hline & 17 & FS2 & Southern Fusong county & Sand \\
\hline & 18 & FS3 & Southern Fusong county & Sand \\
\hline & 19 & FS4 & Southern Fusong county & Loess \\
\hline \multirow[t]{4}{*}{$\mathrm{F}$} & 20 & FS5 & Northern Fusong county & Sand \\
\hline & 21 & FS6 & Northern Fusong county & Sand \\
\hline & 22 & FS7 & Northern Fusong county & Sand \\
\hline & 23 & FS8 & Northern Fusong county & Sand \\
\hline \multirow[t]{4}{*}{$\mathrm{G}$} & 24 & JA1 & Jian county & Loess \\
\hline & 25 & JA2 & Jian county & Sand \\
\hline & 26 & JA3 & Jian county & Sand \\
\hline & 27 & JA4 & Jian county & Sand \\
\hline \multirow[t]{3}{*}{$\mathrm{H}$} & 28 & FS9 & Changbai city & Loess \\
\hline & 29 & FS10 & Changbai city & Sand \\
\hline & 30 & FS11 & Changbai city & Sand \\
\hline
\end{tabular}

${ }^{a}$ Dash (-) indicates a lack of storage medium.
The extracted DNA was quantified using the Qubit 2.0 dsDNA HS Assay Kit, and the DNA used as the template for polymerase chain reaction (PCR) amplifications was selected accordingly. The universal primers (ITS1/2) used with the MiSeq sequencing platform are listed in Table 2 (Bellemain et al. 2010). After two rounds of PCR amplification, the DNA quality was evaluated using the Qubit 2.0 dsDNA HS Assay Kit and the Agilent 2100 Bioanalyzer. A DNA library was constructed with the TruSeq DNA PCR-Free Sample Preparation Kit and then quantified with the Qubit2.0 dsDNA HS Assay Kit and quantitative real-time PCR. The DNA library was then sequenced on a MiSeq $2 \times 300$ platform (paired end, $250 \mathrm{bp}$ ). The sequencing and subsequent analyses were completed by Novogene (Beijing, China).

Data analyses of operational taxonomic units and alpha and beta diversity. Sample sequences were distinguished by DNA barcodes for the raw data. Additionally, the adapters and primer sequences were eliminated from the MiSeq sequences, and the FLASH program (version 1.2.7) was used to assemble the reads of each sample to obtain raw tags (Magoč and Salzberg 2011). Tags were cut, and nonspecific amplified sequences and chimeras were removed to obtain highquality clean data (Caporaso et al. 2010). Using the UPARSE program (version 7.0.1001) (Edgar 2013), the effective tags of all samples were clustered into operational taxonomic units (OTUs) with 97\% identity. The representative sequence in the OTU cluster was then obtained. The OTU species annotations were completed using the BLAST method of the QIIME program (version 1.7.0) (Altschul et al. 1990) and the United database. We chose the most abundant sequence as the representative sequence in the OTU cluster and assessed the diversity of the OTU cluster. On the basis of the species annotation results, we selected the top 10, 50, and 100 species for each sample or grouping at each taxonomic level (phylum, class, order, family, and genus) to measure the relative species abundance. Furthermore, evolutionary classifications and phylogenetic information were used to visualize the relative abundance of species and their proportions at different classification levels.

A sample hierarchical clustering tree diagram was constructed based on the abundance of OTUs. The hierarchical clustering analysis is performed according to the beta diversity distance matrix, and then the tree structure is constructed by using the unweighted pair group method with arithmetic mean (Lozupone and Knight 2005; Lozupone et al. 2010). A nonmetric multidimensional scaling (NMDS) declining dimension map was constructed used the R software Vegan package (Noval Rivas et al. 2013).

Identification of Fusarium and pathogenicity assessment. Twenty-five seeds per group were randomly selected, washed with sterile water, sterilized in $1 \% \mathrm{NaClO}$ for $2 \mathrm{~min}$, and then rinsed twice with sterile water. On average, 8 to 9 seeds were evenly distributed on each of three $9-\mathrm{cm}$ potato dextrose agar (PDA) plates. The plates were incubated in a $25^{\circ} \mathrm{C}$ incubator for 3 to 10 days under a 12 -h light/12-h dark photoperiod. The seed-borne Fusarium species were then isolated.

Morphological characteristics of isolated Fusarium species were observed while referring to Leslie and Summerell (2006). Using rDNA-ITS, TEF- $1 \alpha$, and BT as DNA barcodes, further molecular identifications were completed via the PCR amplification of genomic

Table 2. Details regarding the PCR primers used for species identification and gene sequencing

\begin{tabular}{|c|c|c|c|c|c|}
\hline Gene region & Primer & Direction & $\begin{array}{l}\text { Base size } \\
\quad \text { (bp) }\end{array}$ & $\begin{array}{c}\text { Annealing } \\
\text { temperature }\left({ }^{\circ} \mathbf{C}\right)\end{array}$ & Reference \\
\hline Internal transcribed spacers (ITS1-2) & $\begin{array}{l}\text { ITS5-1737F } \\
\text { ITS2-2043R }\end{array}$ & $\begin{array}{l}\text { Sense } \\
\text { Antisense }\end{array}$ & 250 & 50 & Bellemain et al. (2010) \\
\hline Internal transcribed spacers (ITS) & $\begin{array}{l}\text { ITS4 } \\
\text { ITS5 }\end{array}$ & $\begin{array}{l}\text { Sense } \\
\text { Antisense }\end{array}$ & 550 & 51 & White et al. (1990) \\
\hline $\begin{array}{l}\text { Translation elongation factor 1-alpha } \\
(\mathrm{TEF}-1 \alpha)\end{array}$ & $\begin{array}{l}\text { EF1T } \\
\text { EF2T }\end{array}$ & $\begin{array}{l}\text { Sense } \\
\text { Antisense }\end{array}$ & 650 & 55 & O’Donnell et al. (2000) \\
\hline$\beta$-tubulin (BT) & $\begin{array}{l}\mathrm{T} 1 \\
\mathrm{~T} 2 \\
\beta \mathrm{t} 2 \mathrm{~b}\end{array}$ & $\begin{array}{l}\text { Sense } \\
\text { Antisense } \\
\text { Antisense }\end{array}$ & 500 & 52 & Glass and Donaldson (1995) \\
\hline
\end{tabular}


DNA using the primers listed in Table 2. The PCR amplification conditions were as follows: $94^{\circ} \mathrm{C}$ for $4 \mathrm{~min}$; 35 cycles of $94^{\circ} \mathrm{C}$ for $50 \mathrm{~s}$, annealing temperature $\left(51,55\right.$, or $\left.52^{\circ} \mathrm{C}\right)$ for $50 \mathrm{~s}$, and $72^{\circ} \mathrm{C}$ for $1.5 \mathrm{~min} ; 72^{\circ} \mathrm{C}$ for $10 \mathrm{~min}$; and $4^{\circ} \mathrm{C}$ hold. The amplicons were analyzed by $0.8 \%$ agarose gel electrophoresis in $0.5 \times$ TAE buffer ( $80 \mathrm{~mA}$ and $110 \mathrm{~V}$ for $43 \mathrm{~min}$ ) and then purified using a MinElute PCR Purification Kit (Qiagen, Hilden, Germany). The purified amplicons were sequenced by Sangon Biotech (Shanghai, China). After removing low-quality sequences, a neighbor-joining method was used to construct a phylogenetic tree with the MEGA 7.06 program. The taxonomic status and genetic relationships were then determined.

Three strains of each Fusarium species were randomly selected for pathogenicity tests on seedlings and 2-year-old ginseng plants. Following the published method (Goswami et al. 2008), the radicles ( $2 \mathrm{~cm}$ length) of sprouted seeds were placed on Petri plates $(15 \mathrm{~cm})$ with two pieces of sterile filter paper and then covered with an agar plug (4-mm diameter) containing mycelium of the fungus. The inoculated radicles were evaluated after 3 days. Blank PDA medium plates were used as controls. Five seedlings were used for each isolate and control.

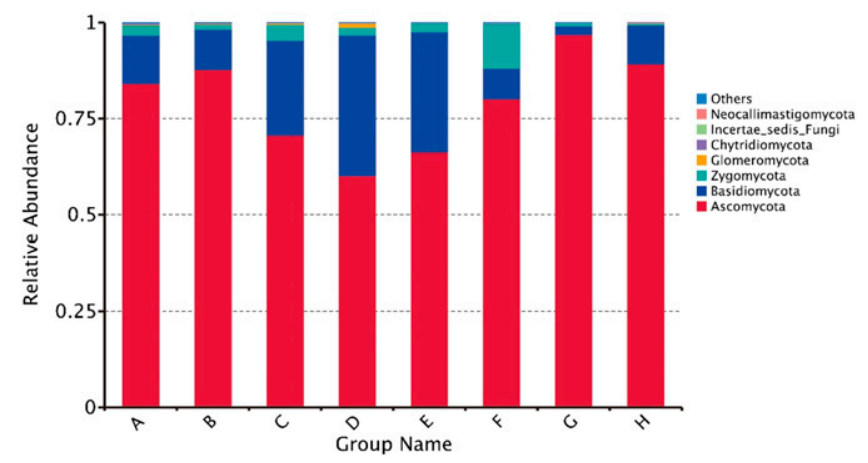

Fig. 1. Comparison of the fungal communities at the phylum level. Groups $A$ to $H$ represent seed samples collected from different regions, which are listed in Table 1. Each color represents a phylum, and the relative abundance of each phylum is indicated as a percentage.
The pathogenicity of the Fusarium isolates on 2-year-old ginseng plants ('Damaya') was evaluated using the method of Song et al. (2014). The tuberous roots were excised and washed, and the root surfaces were wiped with $75 \%$ alcohol. Roots were inoculated by inserting two cut-off pipette tips $(200 \mu \mathrm{l}, \mathrm{BBI})$ with inoculum into the roots. Each pipette tip contained $30 \mu \mathrm{l}$ of spore suspension $\left(1 \times 10^{6} / \mathrm{ml}\right)$, and they were inserted into the root to a depth of $5 \mathrm{~mm}$. Three ginseng roots were inoculated for each Fusarium isolate and for the control treatment, which used sterile water. The samples were then placed in a crisper and incubated at $25^{\circ} \mathrm{C}$ with a 12-h light/ 12-h dark photoperiod and evaluated after 3 days.

\section{Results}

Analysis of the diversity and abundance of fungal communities. On average, 45,676 sequences per sample were obtained as raw data, and 45,545 sequences per sample, with an average read length of $234.93 \mathrm{bp}$, were acquired after the elimination of joint and chimeric sequences. The sequences were divided into seven phyla, 30 classes, 101 orders, 223 families, and 384 genera. Ascomycota and Basidiomycota sequences accounted for 79.43 and $17.01 \%$ of the sequences, respectively.

A taxonomic distribution bar plot revealed the species and their proportions at the phylum level as well as the relative abundance of genera in each sample group. Each group mainly comprised Ascomycota, Basidiomycota, and Zygomycota fungi (Fig. 1). Glomeromycota existed in all groups, and groups $\mathrm{C}$ and $\mathrm{D}$ had a higher proportion, which were 1.01 and $0.32 \%$, respectively. Group G had more Ascomycota fungi, with a content of $96.89 \%$, and less Basidiomycota and Zygomycota fungi, with a content of 2.27 and $0.79 \%$. Group $\mathrm{F}$ had the most Zygomycota fungi, which was $11.67 \%$. The 10 most abundant genera were Penicillium, Fusarium, Pseudogymnoascus, Lecythophora, Debaryomyces, Plectosphaerella, Cephalotrichum, Alternaria, Botrytis, and Discostroma (Fig. $2)$. The 50 most abundant genera (10 unclassified to genus level are combined as "unclassified to genus"), including Fusarium, Alternaria, Nectria, Coniothyrium, Verticillium, Phoma, and Rhizoctonia (Fig. 2), were responsible for the main ginseng diseases, including red-skin or root rot, leaf spot, rusted root disease, dropping-off disease, and other diseases (Lu et al. 2019). Beneficial fungi that exist universally in the environment were also detected, such as some of Penicillium, Debaryomyces, and Trichoderma species. Pathogens,

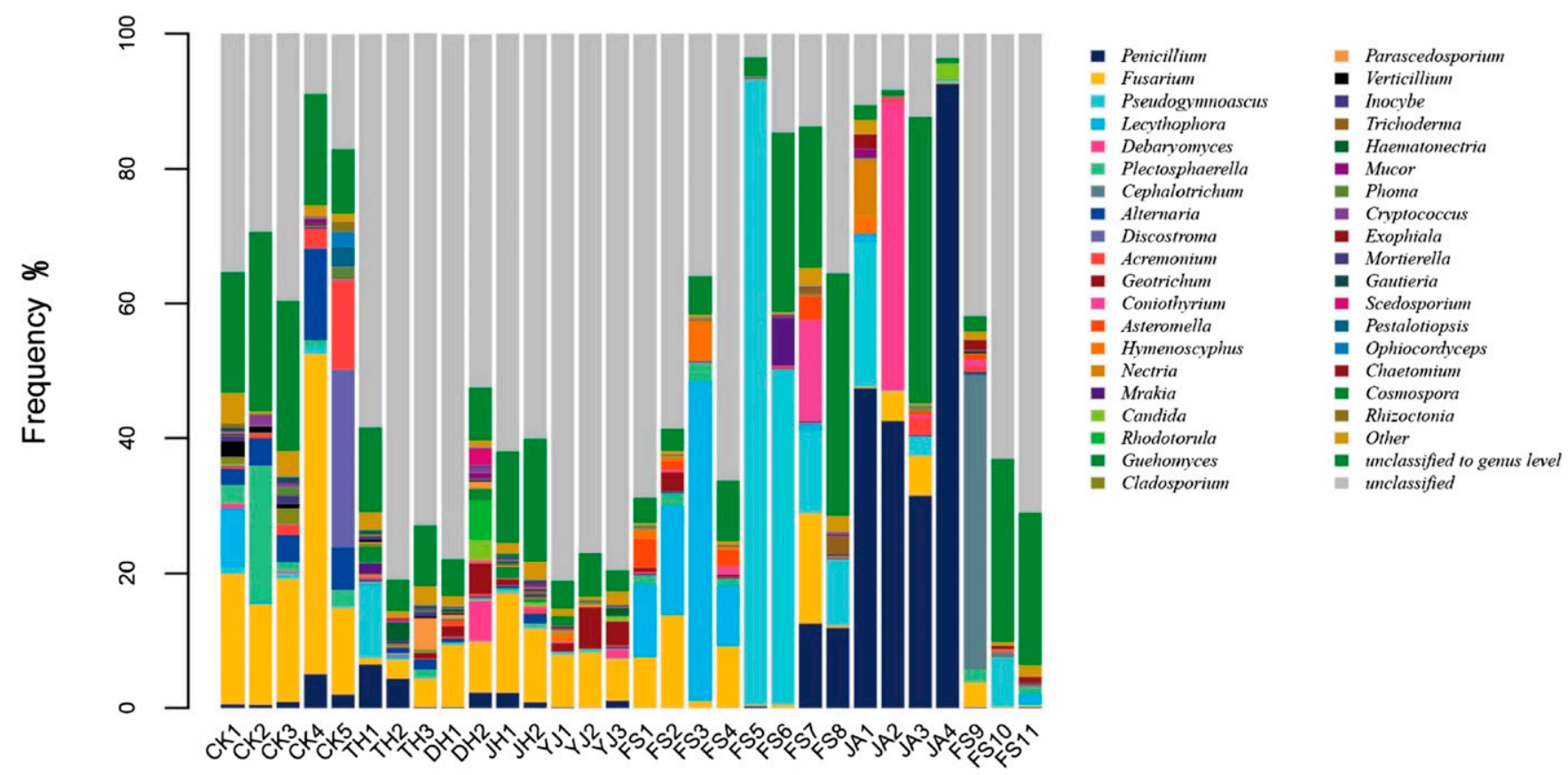

Fig. 2. Comparison of the fungal communities on seed samples at the genus level. Each color represents a genus, and the frequency of each genus represents the relative abundance. 
including a Nectria species causing rusted root disease, $R$. solani causing damping-off, and Alternaria panax causing leaf spot, were also detected.

To further clarify the evolutionary relationships at the genus level, a multiple sequence alignment of the representative sequences for the 100 most abundant genera was completed and integrated with their relative abundance levels (Fig. 3). Of the top 100 species, 96 were Ascomycota (upper right, 68\%) or Basidiomycota (left, 28\%) fungi. Thus, the ginseng seeds were infected primarily by fungi from two phyla, and the 20 most abundant species were Ascomycota fungi. Not including unclassified fungi, Fusarium and Penicillium were the only genera that were detected in all samples at relatively high abundance levels. Fusarium and Penicillium represented 0.22 to $47.5 \%$ and 0.1 to $92.55 \%$, respectively, of the fungi in each sample.
Fusarium species are the main cause of ginseng root rot and were detected in all seed samples regardless of whether the seeds were stored in media. These observations may be useful for developing or improving methods to prevent or treat seed-borne diseases.

Relationships between fungal communities and regions. Similarities and differences among samples can be presented in a hierarchical clustering tree diagram (Fig. 4). Seed-borne fungi from eight different areas formed a cluster. The diversity of seed-borne fungi revealed their region-related characteristics. Samples from the same region were more closely related. However, samples from different but adjacent counties were also closely related, indicating these characteristics were associated with where the seeds originated. A sample clustering tree diagram analysis showed that samples from the same region were clustered in the same branch. The control group did not

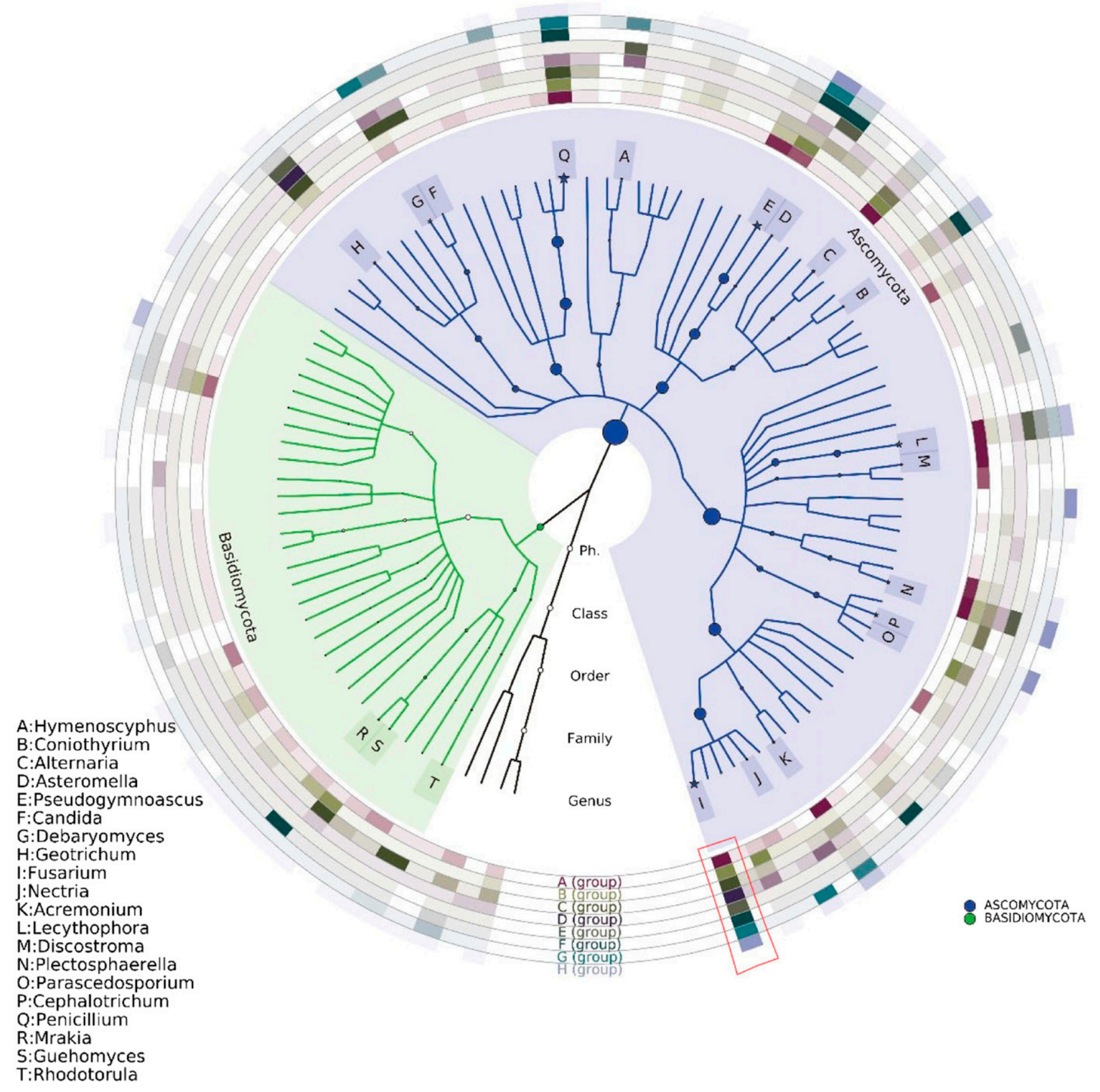

Fig. 3. Visualization of the evolutionary classifications and phylogenetic relationships among the 100 most abundant species. Letters A to T correspond to the 20 most abundant genera (marked with a star), which are indicated by different colors. The size of the circle and star represents the abundance. The outer rings represent a heat map, with each ring corresponding to a group. Individual samples are presented in different colors. The color intensity varies depending on species abundance. 
form two large branches with the other groups but formed an independent branch. The control group samples were taken from different locations but were still clustered in one branch, indicating that the storage medium brings additional fungi to the seeds. We herein describe the fungi on ginseng seeds and the fungi that are transferred from the media to the seeds. The fungi carried by the seeds are more closely related to the region. The clustering of the OTU by geographic group suggested local populations may predominate in certain fields owing to an unknown selective advantage or founder effect. Seeds stored in media contained more pathogenic fungi than seeds that had not been stored in media.

The similarity of the composition of fungal communities between and within groups was assessed using NMDS (Fig. 5). We labeled each group's samples in ellipses. Samples from the same group formed clusters and were assigned to an ellipse. Except for groups $\mathrm{B}$ and $\mathrm{C}$, the other groups were assigned to one region independently, indicating that these samples being divided into one group was supported. Additionally, there was an overlap between groups B and C, indicating that seed-borne fungi from nearby areas were highly

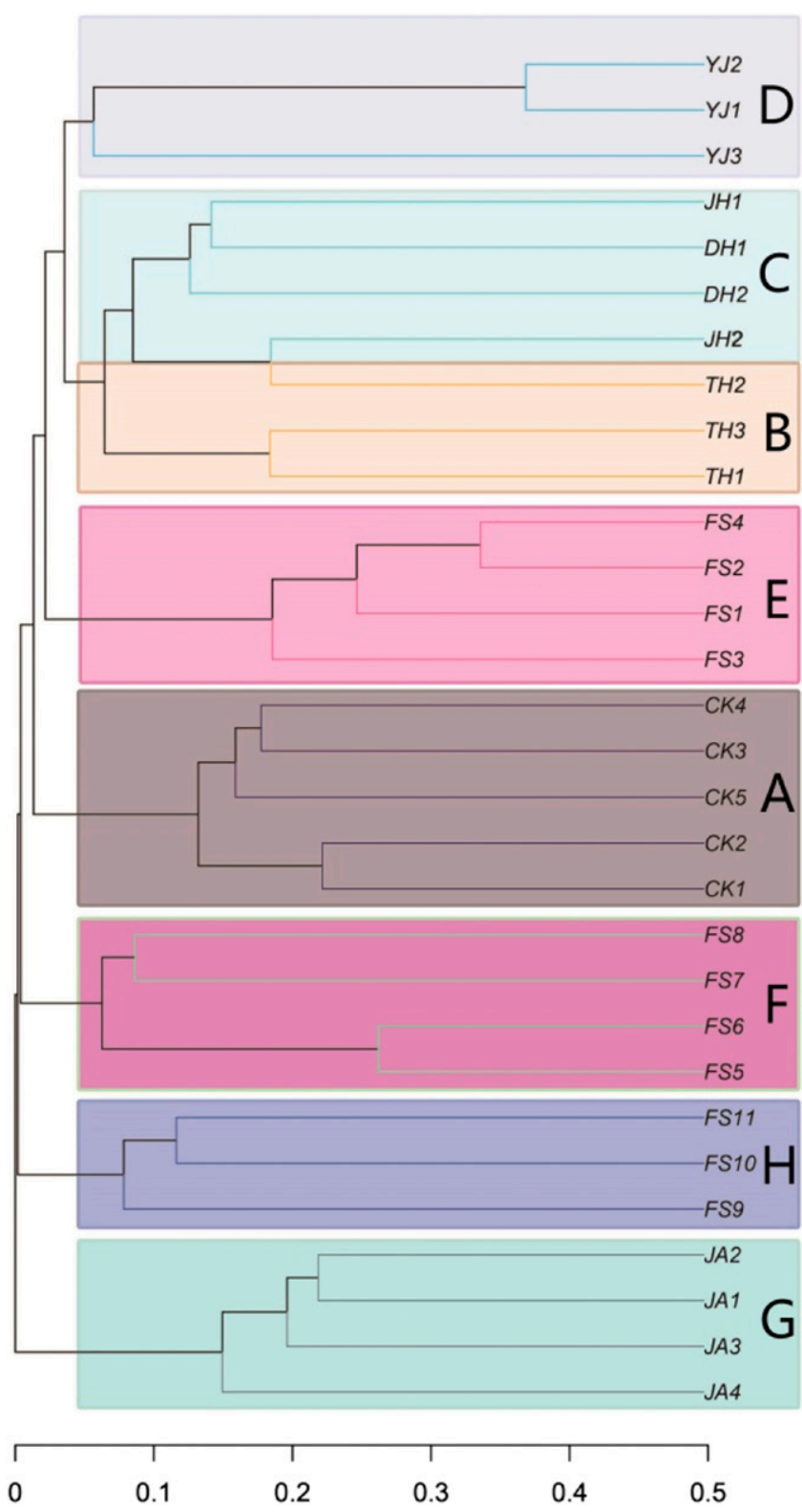

Fig. 4. Sample hierarchical clustering tree diagram based on operational taxonomic units. Branch length represents the distance between samples, with shorter branches for more closely related samples. Samples in the same color belong to the same group. similar. The result was consistent with the hierarchical clustering analysis of OTUs, because the sample TH2 is closely related to groups $\mathrm{B}$ and $\mathrm{C}$. The results confirmed the hierarchical clustering analysis.

Isolation and species confirmation of seed-borne Fusarium species. A total of 518 Fusarium isolates were obtained from 30 samples and then classified into 10 species based on morphology and molecular biology. The 10 species, $F$. solani, $F$. oxysporum, $F$. acuminatum, $F$. proliferatum, $F$. commune, $F$. fujikuroi, $F$. redolens, $F$. avenaceum, $F$. incarnatum, and $F$. cerealis, represented 45.0, $23.4,9.2,4.6,3.2,2.8,2.8,3.2,3.2$, and $2.8 \%$ of the whole community, respectively. Several ginseng root rot-causing Fusarium species were included. Moreover, $F$. solani and $F$. oxysporum were the two most abundant Fusarium species, which was consistent with the identified pathogens causing ginseng root rot during cultivation. GenBank accession numbers were obtained for 30 strains that were randomly selected to construct a phylogenetic tree. The GenBank accession numbers for the ITS, TEF- $1 \alpha$, and BT sequences from each strain are listed in Table 3 . A total of 55 strains were used to construct the phylogenetic tree based on ITS, TEF-1 $\alpha$, and BT sequences (Fig. 6 ). The NCBI database lacks reference genes for $F$. commune because this species was identified relatively recently. The phylogenetic reconstruction revealed 10 main clades $(F$. proliferatum, $F$. fujikuroi, $F$. commune, $F$. oxysporum, $F$. redolens, $F$. cerealis, $F$. incarnatum, $F$. avenaceum, $F$. solani, and $F$. acuminatum). Additionally, selected isolates clustered with reference sequences, with the bootstrap value of the clade exceeding $90 \%$. Moreover, $F$. proliferatum, $F$. fujikuroi, $F$. commune, $F$. redolens, and $F$. oxysporum were located on a large branch, suggesting they are genetically closely related. The clades with $F$. avenaceum and $F$. solani strains exhibited intraspecific diversity. The identified strains were verified based on their morphological characteristics.

Pathogenicity of seed-borne Fusarium species. Pathogenicity was verified based on Koch's postulates. The two inoculation methods used in this study revealed that the 10 Fusarium species recovered from ginseng seeds were all pathogenic to developing seedlings and the roots of 2-year-old plants. Three days after being inoculated with Fusarium according to the first published method, the inoculated areas were reddish brown or transparent, withered, and damaged (Fig. 7A). Control samples grew normally without any symptoms. For the second method of inoculation, the 2-yearold ginseng roots showed vigorous growth of hyphae, and the inoculation site became soft and rotted. Red-brown rotten ginseng root tissue can be seen along the longitudinal section of the inoculation centerline (Fig. 7B and C). Control samples were free of symptoms. The same fungus was reisolated from the infected tissues and confirmed by morphological and molecular methods described previously. Inoculation experiments indicated that seed-borne Fusarium species can infected seedlings and 2-year-old ginseng roots. The fungi used for inoculations considerably inhibited radicle and hypocotyl growth, which may result in large-scale death during the seedling stage.

\section{Discussion}

It is unclear what varieties of ginseng seeds were used in the experiments. Most of the currently grown Chinese cultivars are domesticated varieties, and Damaya ginseng is commercially cultivated. Thus, the correlation between the presence of fungi on ginseng seeds and variety has not been analyzed. During the commercial production of ginseng, farmers usually store the seeds deep underground using nearby media, such as loess and sand, and no other protective measures are taken. Thus, pathogenic fungi can be carried by seeds, which represents a potential threat to ginseng cultivation.

The NMDS was designed to overcome the shortcomings of principal component analyses and principal coordinate analyses to better reflect the nonlinear structure of ecological data. In the NMDS analysis, species information was reflected in a multidimensional space as a formation of dots. Differences among samples between and within groups were indicated by the distance between dots. Thus, samples with a highly similar community structure tend to cluster 
together, in contrast to samples with large differences. Except for groups $\mathrm{B}$ and $\mathrm{C}$, the other group was basically concentrated in one area, and we can still see that samples of each group were clustered together.

An analysis of a combination of molecular characteristics increases the accuracy of fungal identifications, but morphological features are also important aids. For example, $F$. commune is closely related to F. oxysporum, and Skovgaard separated it from the $F$. oxysporum complex in 2003 (Skovgaard et al. 2003). The occasional production of polyphialides in $F$. commune is a unique trait that distinguishes it from the $F$. oxysporum complex. Furthermore, $F$. redolens was once considered to be a variant of $F$. oxysporum and $F$. solani (Gordon 1952). This paper does not find a new species of Fusarium; the morphological features refer to a published description and are similar, so the relevant description is omitted here. ITS recognition is not specific enough to distinguish between closely related species. Consequently, several genes in a joint phylogenetic analysis are required.

Seed-borne pathogens are responsible for many diseases of seeds and seedlings, with infections occurring before or during seed sprouting. Spores can be produced by fungal pathogens (such as Pythium and Fusarium species) in infected host tissues. Some fungi, especially Alternaria species, might be seed-borne pathogens (Punja et al. 2007). Others can be transmitted through straw in the garden or through soil containing infected plant residues. Harvested ginseng seeds require some additional time to completely ripen. Moreover, the seeds may be particularly susceptible to infection during this period. When the endosperm matures, the endocarp cracks, so the endosperm is exposed to the storage medium. Exactly how Fusarium species infect seeds remains unclear. Mycotoxin contamination of oat seeds affected by Fusarium head blight is associated with germination capacity (Tekle et al. 2013). Using scanning electron microscopy to observe the mode of attack used by Fusarium on Bromus tectorum seeds showed that the fungal hyphae first penetrated the caryopsis wall, then entered the embryo, and later ramified throughout the endosperm, completely destroying the seeds (Franke et al. 2014).

Many important ginseng diseases are seed-borne, especially after the seeds are stored in media (Wang 2001). Ginseng seeds may be highly infected by known potentially damaging pathogenic fungi, and the transmission of Fusarium species may be via infected seeds, soil, or both (Ziezold et al. 1998). Park et al. (2012b) reported that the identities of the fungi isolated from different parts of ginseng plants grown in Korea over a 3- or 4-year period were verified. Additionally, others reported that Fusarium species, such as F. redolens, F. cerealis, and $F$. acuminatum, were among the main causes of ginseng root rot (Gao et al. 2014; Guan et al. 2014; Wang et al. 2016). Park et al. (2012a) also observed endophytic fungi on ginseng including $F$. oxysporum whose distribution was dependent on the age of the ginseng plants, similar to other endophytic fungi. Meanwhile, $11 \mathrm{Fusa}$ rium species in the soil were reported by Lee (2004) to be responsible for ginseng root rot, with most of these species highly pathogenic to ginseng. During the commercial production of ginseng, root rot is primarily caused by $F$. solani and $F$. oxysporum (Wang 2001). Many disease-associated pathogens were detected in the top 50 most abundant genera, but in addition to Fusarium, we did not isolate the fungi of these genera and evaluate their pathogenicity. Many pathogens causing ginseng red skin include $F$. acuminatum, $F$. avenaceum, $F$. solani, and $F$. torulosum, and the other three except $F$. torulosum were isolated in this test. Although the test showed the same results as the root rot pathogens, there are many factors causing root rot, and the seeds may be a source of inoculum. However, three types of $F$. circinatum infection have been described (i.e., superficial, active internal, and dormant internal) (Storer et al. 1998). Nevertheless,

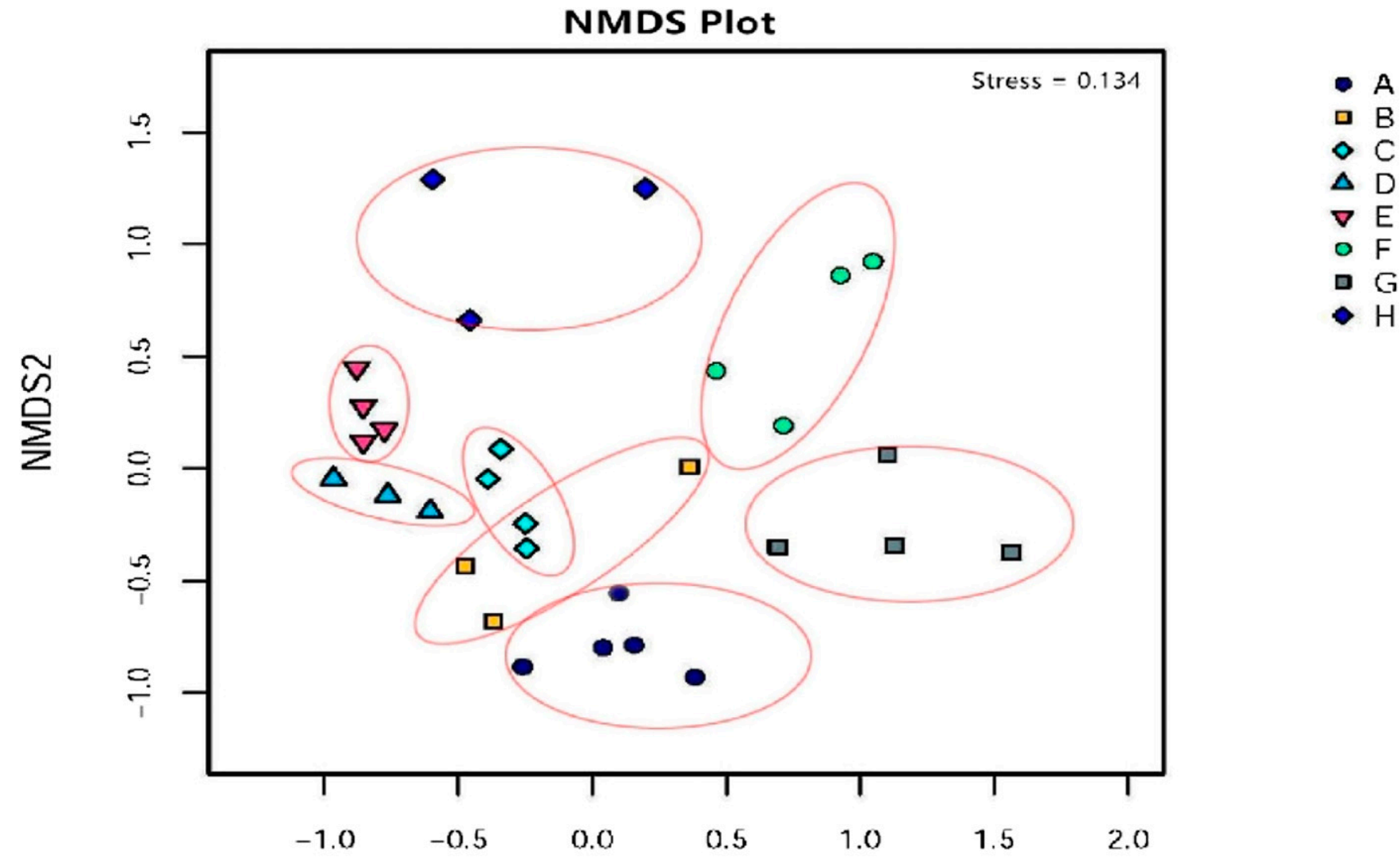

NMDS1

Fig. 5. Nonmetric multidimensional scaling (NMDS) plot of all seed samples. The figure was constructed based on the operational taxonomic unit data. Samples with the same shape and color represent a group. The distance between two points indicates the relationship between samples. 
contaminated seeds may produce more diseased roots than clean seeds (Ziezold et al. 1998). Fusarium species survive by infecting seeds under adverse conditions, entering a dormancy period, and then infecting the seeds when the conditions are appropriate (e.g., during seed germination) (O'Donnell et al. 2000). Fusarium species cannot only affect early seed germination and infect seedlings but also secrete fusaric acid and other toxins (Desjardins et al. 2000). Lee examined 11 Fusarium species (115 isolates) from rotted plants and soil (Lee 2004). The proportion of the isolates represented by $F$. solani (55 isolates) and $F$. oxysporum (35 isolates) was consistent with our results.
Perennial healthy ginseng roots were hard to obtain for pathogenicity testing for the isolates, so we used a visual inoculation protocol of two published methods. One is to assess the pathogenicity of pathogens infecting seedlings, and the other is to assess the pathogenicity of pathogens infecting 2-year-old fresh ginseng roots. This helps to explain the possible threats of pathogens to seedlings and adult plants.

Infections by certain fungal species can inhibit seed germination and facilitate the development of soil-borne diseases, and commercial seeds are likely to introduce diseases into new areas. The prevention of seed-borne diseases can easily be overlooked, and the use of

Table 3. Fusarium species analyzed in this study

\begin{tabular}{|c|c|c|c|c|c|}
\hline \multirow[b]{2}{*}{ Species } & \multirow[b]{2}{*}{ Strain number } & \multirow[b]{2}{*}{ Origin $^{a}$} & \multicolumn{3}{|c|}{ GenBank accessions ${ }^{b}$} \\
\hline & & & ITS & TEF-1 $\alpha$ & BT \\
\hline F. solani & gss53 & China & MH290449 & MH341207 & MH341237 \\
\hline F. solani & gss58 & China & MH290450 & MH341208 & MH341238 \\
\hline F. solani & gss97 & China & MH290451 & MH341209 & MH341239 \\
\hline F. solani & M84 & Poland & KP295502 & KP400749 & KP674305 \\
\hline F. solani & M105A & Poland & KP295505 & KP400752 & KP765701 \\
\hline F. oxysporum & gss100 & China & MH290452 & MH341210 & MH341240 \\
\hline F. oxysporum & gss142 & China & MH290453 & MH341211 & MH341241 \\
\hline F. oxysporum & gss143 & China & MH290454 & MH341212 & MH341242 \\
\hline F. oxysporum & MF5 & Poland & KP719138 & KP710614 & KP710652 \\
\hline F. oxysporum & M228 & Poland & KP264651 & KP400703 & KP674278 \\
\hline F. oxysporum & $\mathrm{Z} 322 \mathrm{C}$ & Poland & KP264657 & KP400710 & KP674232 \\
\hline F. proliferatum & gss170 & China & MH290455 & MH341213 & MH341243 \\
\hline$F$. proliferatum & gss175 & China & MH290456 & MH341214 & MH341244 \\
\hline F. proliferatum & gss176 & China & MH290457 & MH341215 & MH341245 \\
\hline F. proliferatum & 08405 & China & KJ020897 & KJ020896 & KJ127283 \\
\hline F. proliferatum & NRRL 31071 & NRRL & AF291061 & AF291058 & AF291055 \\
\hline F. proliferatum & PT5 & China & KP313248 & KP313249 & KP313250 \\
\hline F. acuminatum & $\operatorname{gss} 151$ & China & МH290459 & MH341216 & MH341246 \\
\hline F. acuminatum & gss 152 & China & MH290458 & MH341217 & MH341247 \\
\hline F. acuminatum & gss163 & China & МH290460 & МH341218 & МH341248 \\
\hline F. acuminatum & IBE000005 & China & EF531248 & EF531236 & EF531243 \\
\hline F. acuminatum & IBE000006 & China & EF531249 & EF531237 & EF531244 \\
\hline F. avenaceum & gss199 & China & MH290476 & MH341234 & MH341264 \\
\hline F. avenaceum & gss202 & China & MH290477 & MH341235 & MH341265 \\
\hline F. avenaceum & gss203 & China & MH290478 & MH341236 & MH341266 \\
\hline F. avenaceum & M127 & Poland & KP265352 & KP674195 & KP710634 \\
\hline F. avenaceum & $\mathrm{Z} 161$ & Poland & KP265369 & KP674212 & KP710650 \\
\hline F. avenaceum & Z337 & Poland & KP295512 & KP400709 & KP674231 \\
\hline F. fujikuroi & gss187 & China & MH290464 & MH341222 & MH341252 \\
\hline F. fujikuroi & gss 190 & China & MH290465 & MH341223 & MH341253 \\
\hline F. fujikuroi & gss191 & China & MH290466 & MH341224 & MH341254 \\
\hline F. fujikuroi & CBS 221.76 & CBS & AB725607 & AB725605 & AB725606 \\
\hline F. incarnatum & gss 207 & China & MH290470 & MH341228 & MH341258 \\
\hline F. incarnatum & gss 210 & China & MH290471 & MH341229 & MH341259 \\
\hline F. incarnatum & gss 211 & China & MH290472 & MH341230 & MH341260 \\
\hline F. incarnatum & NRRL 31160 & NRRL & GQ505696 & GQ915510 & GQ915444 \\
\hline F. cerealis & $\operatorname{gss} 213$ & China & MH290473 & MH341231 & MH341261 \\
\hline F. cerealis & gss 215 & China & MH290474 & MH341232 & MH341262 \\
\hline F. cerealis & $\operatorname{gss} 218$ & China & MH290475 & MH341233 & MH341263 \\
\hline F. cerealis & Z308 & Poland & KP264658 & KP400711 & KP674233 \\
\hline F. cerealis & M237 & Poland & KP264631 & KP400681 & KP765706 \\
\hline F. cerealis & S346 & Poland & KP264665 & KP400718 & KP674242 \\
\hline F. cerealis & NRRL 25419 & NRRL & AF006340 & AF212465 & AF006360 \\
\hline F. cerealis & NRRL 13721 & NRRL & U85534 & AF212464 & U85568 \\
\hline F. cerealis & NRRL 25805 & NRRL & AF006341 & AF212466 & AF006361 \\
\hline F. commune & gss 180 & China & MH290461 & MH341219 & MH341249 \\
\hline F. commune & $\operatorname{gss} 182$ & China & MH290462 & MH341220 & MH341250 \\
\hline F. commune & gss 183 & China & MH290463 & MH341221 & MH341251 \\
\hline F. redolens & gss193 & China & MH290467 & МH341225 & MH341255 \\
\hline F. redolens & gss196 & China & MH290468 & MH341226 & MH341256 \\
\hline F. redolens & gss198 & China & MH290469 & MH341227 & MH341257 \\
\hline F. redolens & M119 & Poland & KP265347 & KP674190 & KP710629 \\
\hline
\end{tabular}

a NRRL = Agricultural Research Service Culture Collection, Northern Regional Research Laboratory, U.S.A.; and CBS = culture collection of the Centraalbureau voor Schimmelcultures, Fungal Biodiversity Centre, Utrecht, The Netherlands.

${ }^{\mathrm{b}}$ ITS $=$ internal transcribed spacer region; TEF-1 $\alpha=$ translation elongation factor 1 -alpha ; and $\mathrm{BT}=\beta$-tubulin . 
chemical and biological control agents requires an understanding of the pathogens carried by the seeds. This study confirms that $F$. solani and $F$. oxysporum are the dominant fungi in ginseng seeds and that $F$. commune and $F$. redolens are closely related to $F$. oxysporum. Thus, these should be the main targets when developing methods for preventing or controlling ginseng diseases. Seed-borne pathogens are responsible for diseases that decrease ginseng production, yet they are often ignored. Thus, identifying and characterizing seed-borne pathogens is an important part of comprehensive preventative and curative strategies designed to control seed-borne diseases.

We applied molecular analyses to identify the fungi carried on ginseng seeds and confirmed the diversity of the fungi on the seed via the sequencing of amplicons. The identified fungi included many species responsible for the major ginseng diseases. Additionally, our hierarchical clustering tree diagram confirmed that the fungi carried by ginseng seeds were closely related to the region from which the seeds were

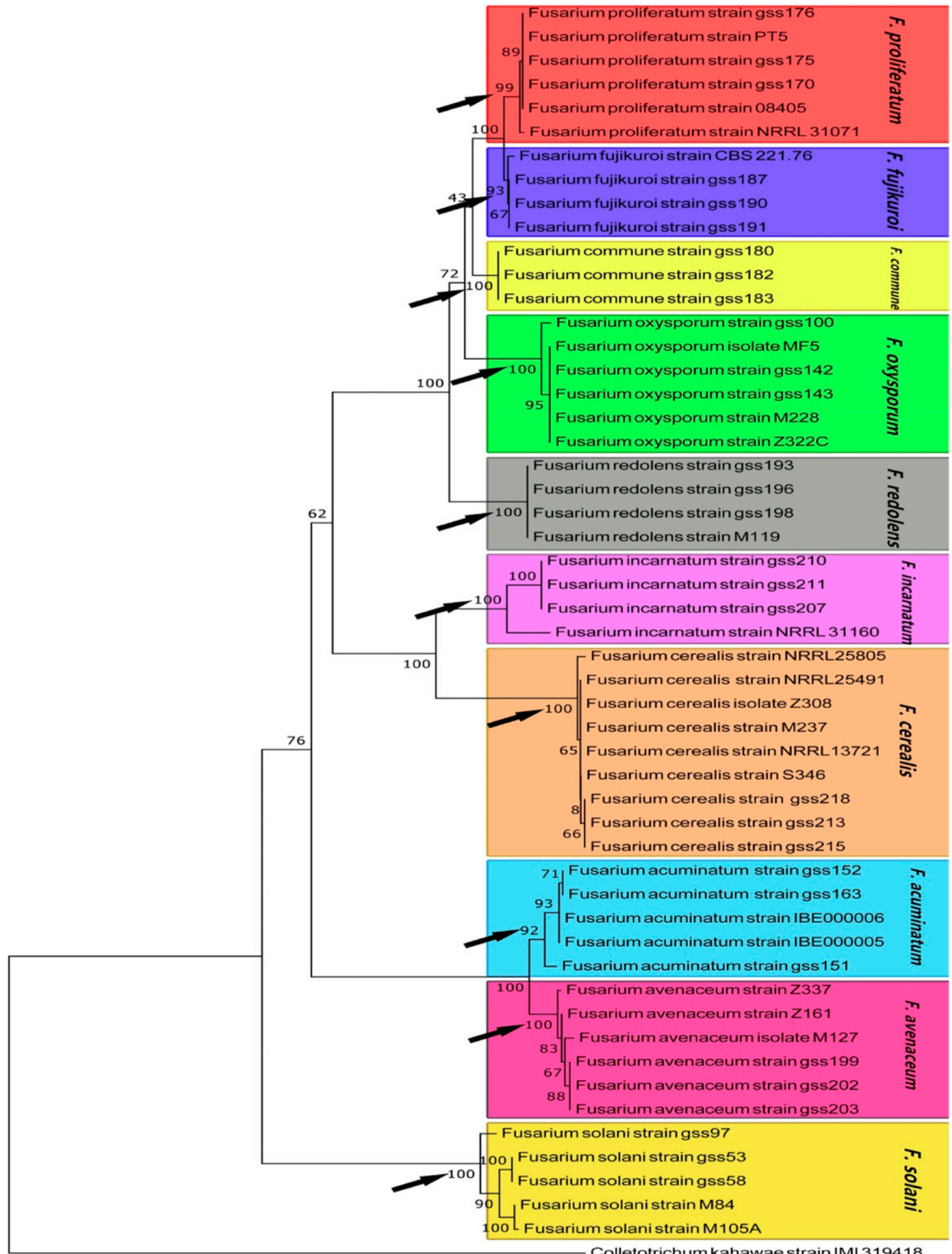

Colletotrichum kahawae strain IMI 319418

$$
\longmapsto 0.020
$$

Fig. 6. Phylogenetic tree based on the combined internal transcribed spacer, translation elongation factor 1 -alpha, and $\beta$-tubulin gene sequences constructed using the neighborjoining method of the MEGA 7.06 program. IMI 319418 was used as an outgroup. 

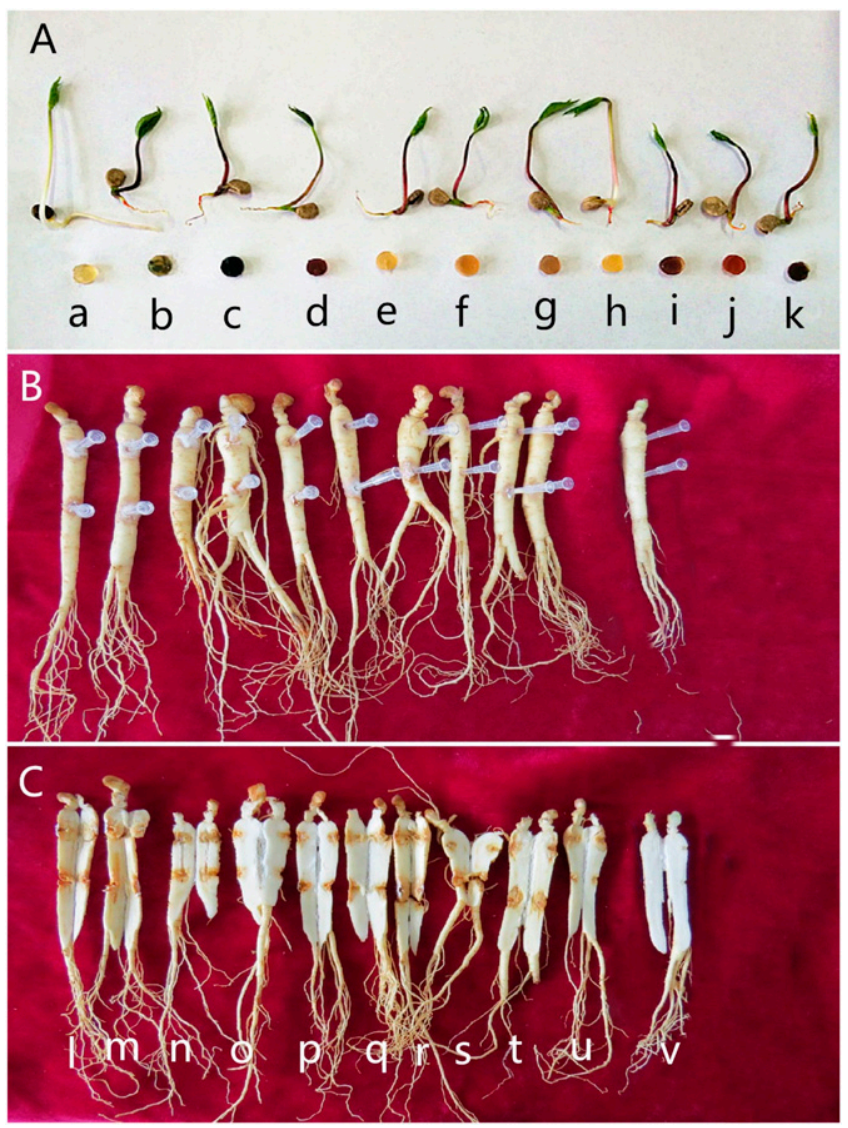

Fig. 7. Symptoms of seedlings and 2-year-old ginseng roots inoculated with Fusarium spp. A, Symptoms of diseased seedlings inoculated with mycelial plugs: $a=$ control; and $\mathrm{b}$ to $\mathrm{k}=F$. solani, $F$. oxysporum, $F$. acuminatum, $F$. proliferatum, $F$. commune, $F$. fujikuroi, F. redolens, F. avenaceum, F. incarnatum, and F. cerealis. B, Symptoms of diseased seeds inoculated with a spore suspension. $C$, Longitudinal section of the inoculation centerline: $v=$ control; and I to $u=F$. solani, $F$. oxysporum, $F$. acuminatum, F. proliferatum, F. commune, F. fujikuroi, F. redolens, $F$. avenaceum, $F$. incarnatum, and $F$. cerealis.

collected and the medium in which the seeds were stored. Highthroughput sequencing data also indicated that Fusarium species were present in all analyzed seeds. We isolated and identified 10 Fusarium species based on morphological and molecular characteristics. Moreover, we analyzed the correlation between seed-borne fungi and the main pathogens of ginseng and also confirmed that the seed-borne Fusarium species could infect ginseng seedlings and 2-year-old plants. The data presented herein may be useful for developing new methods to prevent or treat seed-borne ginseng diseases, with potential implications for improving ginseng yield and quality.

\section{Literature Cited}

Altschul, S. F., Gish, W., Miller, W., Myers, E. W., and Lipman, D. J. 1990. Basic local alignment search tool. J. Mol. Biol. 215:403-410.

Bellemain, E., Carlsen, T., Brochmann, C., Coissac, E., Taberlet, P., and Kauserud, H. 2010. ITS as an environmental DNA barcode for fungi: An in silico approach reveals potential PCR biases. BMC Microbiol. 10:189-197.

Bunkina, M. I. A. 1960. Analysis of ginseng seed for fungal infection and presowing treatment. Mater Izuch Zhenshenya Limonnika. 4:163-170.

Caporaso, J. G., Kuczynski, J., Stombaugh, J., Bittinger, K., Bushman, F. D., Costello, E. K., Fierer, N., Peña, A., Goodrich, G., Gordon, J. K., Huttley, J. I., Kelley, G. A., Knights, S. T., Koenig, D., Ley, J. E., Lozupone, R. E., Mc, C. A., Donald, D., Muegge, B. D., Pirrung, M., Reeder, J., Sevinsky, J. R., Turnbaugh, P. J., Walters, W. A., Widmann, J., Yatsunenko, T., Zaneveld, J., and Knight, R. 2010. QIIME allows analysis of high-throughput community sequencing data. Nat. Methods 7:335-336.

Desjardins, A. E., Manandhar, H. K., Plattner, R. D., Manandhar, G. G., Poling, S. M., and Maragos, C. M. 2000. Fusarium species from Nepalese rice and production of mycotoxins and gibberellic acid by selected species. Appl. Environ. Microbiol. 66:1020-1025.

Edgar, R. C. 2013. UPARSE: Highly accurate OTU sequences from microbial amplicon reads. Nat. Methods 10:996-998.
Franke, J. L., Geary, B., and Meyer, S. E. 2014. Identification of the infection route of a Fusarium seed pathogen into nondormant Bromus tectorum seeds. Phytopathology 104:1306-1313.

Gao, J., Wang, Y., Guan, Y. M., and Chen, C. Q. 2014. Fusarium cerealis, a new pathogen causing ginseng (Panax ginseng) root rot in China. Plant Dis. 98: 1433.

Geiser, D. M., Del Mar Jiménez-gasco, M., Gasco, J., Kang, S., Makalowska, I., Veeraraghavan, N., Ward, T. J., Zhang, N., Kuldau, G. A., and O'Donnell, K. 2004. FUSARIUM-ID v. 1.0: A DNA sequence database for identifying Fusarium. Eur. J. Plant Pathol. 110:473-479.

Glass, N. L., and Donaldson, G. C. 1995. Development of primer sets designed for use with the PCR to amplify conserved genes from filamentous ascomycetes. Appl. Environ. Microbiol. 61:1323-1330.

Gordon, W. L. 1952. The occurrence of Fusarium species in Canada: II. Prevalence and taxonomy of Fusarium species in cereal seed. Can. J. Bot. 30:209-251.

Goswami, R. S., Dong, Y. H., and Punja, Z. K. 2008. Host range and mycotoxin production by Fusarium equiseti isolates originating from ginseng fields. Can. J. Plant Pathol. 30:155-160.

Guan, Y. M., Lu, B. H., Wang, Y., Gao, J., and Wu, L. J. 2014. First report of root rot caused by Fusarium redolens on ginseng (Panax ginseng) in Jilin province of China. Plant Dis. 98:844.

Guan, Y. M., Yan, M. X., Wang, Y. P., Zhang, Y. Y., and Wang, Z. Q. 2010 Infection detection of ginseng seed and disinfection effect of fungicides. Seed. 29:32-34

Howard, R. J., Garland, J. A., and Seaman, W. L. 1994. Diseases and Pests of Vegetable Crops in Canada: An Illustrated Compendium. Canadian Phytopathological Society, Ottawa, ON.

Kim, M. J., Shim, C. K., Kim, Y. K., Hong, S. J., Park, J. H., Han, E. J., and Kim, S. C. 2017. Enhancement of seed dehiscence by seed treatment with Talaromyces flavus GG01 and GG04 in ginseng (Panax ginseng). Plant Pathol. J. 33:1-8.

Lee, J. C., Chung, Y. R., and Ohh, H. P. S. H. 1981. Influence of seed dressing with Captan WP on the dehiscence of Panax ginseng seeds. Korean J. Ginseng Sci. 28:262-266.

Lee, S. G. 2004. Fusarium species associated with ginseng (Panax ginseng) and their role in the root-rot of ginseng plant. Res. Plant Dis. 10:248-259.

Leslie, J. F., and Summerell, B. A. 2006. The Fusarium Laboratory Manual. Blackwell Publishing, Ames, IA.

Lozupone, C., and Knight, R. 2005. UniFrac: A new phylogenetic method for comparing microbial communities. Appl. Environ. Microbiol. 71:8228-8235.

Lozupone, C., Lladser, M. E., Knights, D., Stombaugh, J., and Knight, R. 2010. UniFrac: An effective distance metric for microbial community comparison. ISME J. 5:169-172.

Lu, X. H., Zhang, X. M., Jiao, X, L., Hao, J. J., Zhang, X. S., Luo, Y. and Gao, W. W. 2019. Taxonomy of fungal complex causing red-skin root of Panax ginseng in China. J. Ginseng. Res. doi: doi:10.1016/j.jgr.2019.01.006.

Magoč, T., and Salzberg, S. L. 2011. FLASH: Fast length adjustment of short reads to improve genome assemblies. Bioinformatics 27:2957-2963.

Noval Rivas, M. N., Burton, O. T., Wise, P., Zhang, Y. Q., Hobson, S. A., Lloret M. G., Chehoud, C., Kuczynski, J., DeSantis, T., and Warrington, J. 2013. A microbiota signature associated with experimental food allergy promotes allergic sensitization and anaphylaxis. J. Allergy Clin. Immunol. 131:201-212.

O’Donnell, K., Kistler, H. C., Tacke, B. K., and Casper, H. H. 2000. Gene genealogies reveal global phylogeographic structure and reproductive isolation among lineages of Fusarium graminearum, the fungus causing wheat scab. Proc. Natl. Acad. Sci. U.S.A. 97:7905-7910.

Park, Y. H., Kim, Y. C., Park, S. U., Lim, H. S., Kim, J. B., Cho, B. K., and Bae, H. 2012a. Age-dependent distribution of fungal endophytes in Panax ginseng roots cultivated in Korea. J. Ginseng Res. 36:327-333.

Park, Y. H., Lee, S. G., Ahn, D. J., Kwon, T. R., Park, S. U., Lim, H. S., and Bae, H. 2012b. Diversity of fungal endophytes in various tissues of Panax ginseng Meyer cultivated in Korea. J. Ginseng Res. 36:211-217.

Punja, Z. K. 1997. Fungal pathogens of American ginseng (Panax quinquefolium) in British Columbia. Can. J. Plant Pathol. 19:301-306.

Punja, Z. K., Wan, A., Goswami, R. S., Verma, N., Rahman, M., Barasubiye, T., Seifert, K. A., and Lévesque, C. A. 2007. Diversity of Fusarium species associated with discolored ginseng roots in British Columbia. Can. J. Plant Pathol. 29:340-353.

Punja, Z. K., Wan, A., Rahman, M., Goswami, R. S., Barasubiye, T., Seifert, K. A., and Lévesque, C. A. 2008. Growth, population dynamics, and diversity of Fusarium equiseti in ginseng fields. Eur. J. Plant Pathol. 121:173-184.

Reeleder, R. D., and Fisher, P. 1995. Diseases of Ginseng. Ministry of Agriculture, Food \& Rural Affairs, Ottawa, ON, Canada.

Skovgaard, K., Rosendahl, S., O’Donnell, K., and Nirenberg, H. I. 2003. Fusarium commune is a new species identified by morphological and molecular phylogenetic data. Mycologia 95:630-636.

Song, J. Y., Seo, M. W., Kim, S. L., Nam, M. H., Lim, H. S., and Kim, H. G. 2014 Genetic diversity and pathogenicity of Cylindrocarpon destructans isolates obtained from Korean Panax ginseng. Mycobiology 42:174-180.

Storer, A. J., Gordon, T. R., and Clark, S. L. 1998. Association of the pitch canker fungus, Fusarium subglutinans f. sp. pini with Monterey pine seeds and seedlings in California. Plant Pathol. 47:649-656. 
Tekle, S., Skinnes, H., and Bjornstad, A. 2013. The germination problem of oat seed lots affected by Fusarium head blight. Eur. J. Plant Pathol. 135:147-158.

Wang, T. S. 2001. China Ginseng. Liaoning Science and Technology Press, Liaoning, China.

Wang, Y., Guan, Y. M., Lu, B. H., and Gao, J. 2016. First report of ginseng (Panax ginseng) root rot caused by Fusarium acuminatum in China. Plant Dis. 100:525.

White, T. J., Bruns, T., Lee, S., and Taylor, J. W. 1990. Amplification and direct sequencing of fungal ribosomal RNA genes for phylogenetics. Pages 315-322 in: PCR Protocols: A Guide to Methods and Applications.M. A. Innis, D. H. Gelfand, J. J. Sninsky, and T. J. White, eds. Academic Press, San Diego, CA. Zhang, T. Y., Zhang, Y., Chen, W. Q., and Zhang, C. W. 1989. An analysis of seedborne fungi of imported seeds of Panax quinquefolium. Acta Phytopathologica Sin. 19:134.

Ziezold, A. M., Reeleder, R. D., Hall, R., and Proctor, J. T. A. 1998. Seedborne fungi and fungicide seed treatment of ginseng. J. Ginseng Res. 22:229-236 